\title{
Ward identities for transport of classical waves in disordered media
}

\author{
H. T. Nieh, ${ }^{*}$ Liu Chen, ${ }^{\dagger}$ and Ping Sheng \\ The Hong Kong University of Science and Technology, Clear Water Bay, Kowloon, Hong Kong
}

(Received 25 June 1997)

\begin{abstract}
Using field-theoretic formalism, we derive Ward identities for the diffusive transport of classical waves in disordered media of a quite general nature. We consider three cases: the scalar wave, the elastic wave, and the electromagnetic wave. For classical waves, it is the energy conservation that underlies the Ward identities, in contrast to the case of electronic systems, for which the Ward identity is a mathematical statement of charge conservation. For the three cases of classical waves, the Ward identities are of the same general structure, which differs from the electronic Ward identity and reflects the fact that energy transport, instead of charge transport, is accounted for in classical wave systems. The general structure of the Ward identities is seen to be independent of the details of the wave equations. [S1063-651X(97)06611-7]

PACS number(s): 03.40.Kf, 41.20.Jb, 62.30.+d, 03.70.+k
\end{abstract}

\section{INTRODUCTION}

Conservation laws are basic to the dynamical properties of physical processes. Ward identities corresponding to the conservation laws are explicit expressions that are essential to a theoretical description of dynamical processes. In electronic systems, the often quoted Ward identity [1] is a statement of charge conservation. For classical waves propagating in inhomogeneous media, however, it is the Ward identities for energy conservation that underlie the theoretical description of wave transport behavior. There seems to be general confusion in the literature [2,3] concerning the derivation as well as the exact form of the Ward identities for the diffusive transport of various classical waves. The Ward identity given by Barabanenov and Ozrin [4] for the scalar wave, for example, is known to be in dispute [2,3]. In the case of an electromagnetic (EM) wave in inhomogeneous media, a Ward identity for constant magnetic permeability has also been presented in Ref. [5]. We shall comment on the results of these works later, and note here that our results differ from them $[4,5]$.

There exists a very general and well-established quantum field-theoretic method for deriving Ward identities from the conservation laws. This is the method due to Takahashi [6], who generalized the original Ward identity [7] for current conservation in quantum electrodynamics. In this paper, although the waves we consider are classical fields, welldeveloped algorithms for quantum fields can nevertheless be borrowed to derive the energy Ward identities for the classical waves, treating the media phenomenologically in terms of classical characterizations, e.g., the dielectric constant $\varepsilon(\mathbf{x})$ and magnetic permeability $\mu(\mathbf{x})$ in the case of the electromagnetic wave. It is well known that the classical fields are the limiting cases of their respective quantum fields [8]. In more exact terminology, the classical fields are quantum fields at the so-called tree, or nonloop, level. It is at this level

\footnotetext{
*Permanent address: Center for Advanced Study, Tsinghua University, Beijing, China.

†Permanent address: Department of Physics, University of California at Irvine, Irvine, CA.
}

that our results are to be understood; there is no quantum effect involved. In this sense the quantum field-theoretic approach is used here only as a powerful "accounting"' algorithm for deriving the desired results.

As one important application of the basic Ward identity, we develop further, with the help of an integral equation whose validity is on the same footing as the Bethe-Salpeter equation, another version of the Ward identity for the description of multiply scattered waves transport in inhomogeneous media [9]. This version of the Ward identity, which we denote the diffusive Ward identity, is known in particular to be relevant for the determination of the energy transport velocity in the wave diffusion constant [10]. We shall see that it is the energy conservation law in the case of the classical waves, in contrast to the charge conservation law in the case of the Schrödinger waves (electronic systems), that is responsible for the interesting multiple scattering effects exhibited by classical waves in disordered media [10].

The classical waves we consider are the scalar wave, the elastic wave, and the electromagnetic wave. Although the case of the elastic wave has been discussed elsewhere [11], it is included here for completeness.

The approach we adopt, which is imbedded in the formalism of Lagrangian field theories, is very general in nature, and can be uniformly applied to all classical waves in inhomogeneous media of considerable complexity and generality. In all the cases we consider, whether it is the scalar wave, the elastic wave, or the electromagnetic wave, the diffusive Ward identities are of the same general structure, i.e., that for the energy conservation. One point we would like to emphasize, a point which will become clear from the derivations, is that this general structure is not influenced by the details of the various wave equations, as is widely believed.

\section{LAGRANGIANS FOR CLASSICAL WAVES}

The starting point of most works on classical waves are based on the wave equations. However, the formalism of Lagrangian field theory provides a more systematic framework for the consideration of many formal aspects of the classical wave systems. The quantum version of this formalism, namely, quantum field theory, is known to have been successfully applied to fundamental theories, such as the 
quantum electrodynamics. This algorithm can be usefully employed in treating certain theoretical aspects of the classical waves. Below, we use it to derive the Ward identity as a mathematical statement of the energy conservation.

The starting point of our approach is the Lagrangian density. It gives rise to the desired wave equation as a result of the action principle, and conservation laws as consequences of the symmetry properties of the Lagrangian. Below we list the Lagrangian densities for the scalar wave, the elastic wave, and the electromagnetic wave.

\section{Scalar wave}

There are two versions of the wave equation for the scalar wave amplitude $\phi(\mathbf{x}, t)$ :

$$
\begin{gathered}
{\left[\varepsilon(\mathbf{x}) \partial_{t}^{2}-\nabla^{2}\right] \phi(\mathbf{x}, t)=0,} \\
{\left[\partial_{t}^{2}-\nabla \cdot \frac{1}{\varepsilon(\mathbf{x})} \nabla\right] \phi(\mathbf{x}, t)=0 .}
\end{gathered}
$$

For Eq. (1), the Lagrangian density is

$$
L^{(\mathrm{S} 1)}=\frac{1}{2}\left[\varepsilon(\mathbf{x}) \partial_{t} \phi \partial_{t} \phi-\nabla \phi \cdot \nabla \phi\right],
$$

while, for Eq. (2), it is

$$
L^{(\mathrm{S} 2)}=\frac{1}{2}\left[\partial_{t} \phi \partial_{t} \phi-\frac{1}{\varepsilon(\mathbf{x})} \boldsymbol{\nabla} \phi \cdot \nabla \phi\right] .
$$

\section{Elastic wave}

Elastic waves are vector in nature. The wave equation for locally isotropic but inhomogeneous media is of the form

$$
\rho \ddot{s}_{j}-\partial_{i}\left[\lambda(\boldsymbol{\nabla} \cdot \mathbf{s}) \delta_{i j}+\mu\left(\partial_{i} s_{j}+\partial_{j} s_{i}\right)\right]=0,
$$

where $s_{i}(\mathbf{x}, t)$ is the displacement vector, $\rho(\mathbf{x})$ the mass density, $\lambda(\mathbf{x})$ the longitudinal Lame's (elastic) constant, and $\mu(\mathbf{x})$ the shear modulus. The parametric functions $\rho, \lambda$, and $\mu$ are position dependent, but assumed to be time independent. The Lagrangian density for the elastic wave equation (3) is

$$
L^{(\mathrm{EL})}=\frac{1}{2}\left[\rho(\dot{\mathbf{s}})^{2}-\lambda\left(\operatorname{Tr} \varepsilon_{i j}\right)^{2}-2 \mu \varepsilon_{i j} \varepsilon_{j i}\right],
$$

where the strain tensor is given by

$$
\varepsilon_{i j}=\frac{1}{2}\left(\partial_{i} s_{j}+\partial_{j} s_{i}\right) .
$$

\section{Electromagnetic wave}

The electromagnetic wave equations are the Maxwell equations. We choose to use the four-dimensional notations $x^{\mu}=(t, \mathbf{x})$, where $\mu=(0,1,2,3)$, and the metric tensor with signature

$$
g^{\mu \nu}=g_{\mu \nu}=(1,-1,-1,-1) .
$$

In addition, Greek subscripts (superscripts) denote fourvector indices $(0,1,2,3)$, and Roman subscripts (superscripts the 3 -vector indices. The four-potential is denoted by $A^{\mu}$, and the field strength tensor by

$$
F^{\mu \nu}=\partial^{\mu} A^{\nu}-\partial^{\nu} A^{\mu} .
$$

For a static inhomogeneous medium with dielectric coefficient $\varepsilon(\mathbf{x})$ and magnetic permeability $\mu(\mathbf{x})$, we define

$$
\begin{aligned}
& H^{0 k}=\varepsilon F^{0 k}, \\
& H^{k 1}=\mu F^{k 1} .
\end{aligned}
$$

The source-free Maxwell equations can be written as

$$
\begin{gathered}
\partial_{k} H^{k 0}=0, \\
\partial_{0} H^{01}+\partial_{k} H^{k 1}=0,
\end{gathered}
$$

or, in four-dimensional notation,

$$
\partial_{\mu} H^{\mu \nu}=0 .
$$

The Lagrangian density that yields Eq. (9) is

$$
L^{(\mathrm{EM})}=-\frac{1}{4} H^{\mu \nu} F_{\mu \nu} .
$$

\section{SCALAR WAVE CASE}

The case of the scalar wave is the simplest of the three. Since the train of considerations is very similar in all the three cases, we treat the case of the scalar wave in detail to fully illustrate the major points of our derivation.

There are two versions of the scalar wave equation. We consider the version corresponding to

$$
\left[\varepsilon(\mathbf{x}) \partial_{t}^{2}-\nabla^{2}\right] \phi(\mathbf{x}, t)=0,
$$

with the Lagrangian density given by

$$
L^{(\mathrm{S} 1)}=\frac{1}{2}\left[\varepsilon(\mathbf{x}) \partial_{t} \phi \partial_{t} \phi-\nabla \phi \cdot \nabla \phi\right] .
$$

Known as Noether's theorem, the symmetry properties of the Lagrangian density directly imply the corresponding local conservation laws. Because of the coordinate dependence of $\varepsilon(\mathbf{x})$ in Eq. (S1), the Lagrangian density is not invariant under spatial translations. As a consequence, wave momentum is not conserved; momentum can be interchanged between wave and medium. However, the invariance property of Eq. (S1) under time translation leads to the conservation of the wave energy. There is a well-established procedure of finding the expression for the energy-momentum tensor [12], which, for the one-component scalar wave $\phi$, is given by

$$
T_{\mu \nu}=\frac{\partial L}{\partial\left(\partial^{\mu} \phi\right)} \frac{\partial \phi}{\partial x^{\nu}}-g_{\mu \nu} L .
$$

While the energy density is $T_{00}$, the momentum density or the Poynting vector is $T_{k 0}$. (We note that $T_{k 0} \neq T_{0 k}$ in nonuniform media as in our present case.) Explicitly, we have

$$
\begin{gathered}
T_{00}=\frac{1}{2}(\varepsilon \dot{\phi} \dot{\phi}+\nabla \phi \cdot \nabla \phi), \\
T_{k 0}=\partial_{k} \phi \dot{\phi} .
\end{gathered}
$$

The local energy conservation law that follows from Nöther's theorem is

$$
\partial^{\mu} T_{\mu 0}=\partial_{\mu} T^{\mu 0}=0,
$$

which can be explicitly verified by using wave equation (1). 
Using the formalism of quantum field theory, we quantize the wave system by imposing equal-time commutation relations on the cannonically conjugate variables. Conjugate to the field $\phi(\mathbf{x}, t)$ is the generalized momentum variable $\pi$, given by

$$
\pi(\mathbf{x}, t)=\frac{\partial L}{\partial \dot{\phi}}=\varepsilon(\mathbf{x}) \dot{\phi}(\mathbf{x}, t)
$$

The quantization conditions are

$$
\begin{gathered}
{[\phi(\mathbf{x}, t), \pi(\mathbf{y}, t)]=i \delta(\mathbf{x}-\mathbf{y}),} \\
{[\phi(\mathbf{x}, t), \phi(\mathbf{y}, t)]=0,} \\
{[\pi(\mathbf{x}, t), \pi(\mathbf{y}, t)]=0 .}
\end{gathered}
$$

We recall that the $\varepsilon$ in Eq. (14) is a numerical function, not a quantum operator.

We define the propagator function $G\left(\mathbf{x}, t ; \mathbf{x}^{\prime}, t^{\prime}\right)$ by the "vacuum" expectation value of the time-ordered product

$$
G\left(\mathbf{x}, t ; \mathbf{x}^{\prime}, t^{\prime}\right)=i\left\langle 0\left|T\left\{\phi(\mathbf{x}, t) \phi\left(\mathbf{x}^{\prime}, t^{\prime}\right)\right\}\right| 0\right\rangle .
$$

Here $|0\rangle$, normalized to 1 , represents the ground state of the system, which is not translationally invariant in our present case of inhomogeneous media, and $T$ denotes time ordering according to the definition

$$
\begin{aligned}
T\left\{\phi(\mathbf{x}, t) \phi\left(\mathbf{x}^{\prime}, t^{\prime}\right)\right\} \equiv & \theta\left(t-t^{\prime}\right) \phi(\mathbf{x}, t) \phi\left(\mathbf{x}^{\prime}, t\right)+\phi\left(t^{\prime}-t\right) \\
& \times \phi\left(\mathbf{x}^{\prime}, t^{\prime}\right) \phi(\mathbf{x}, t),
\end{aligned}
$$

where $\theta(t)$ is the step function: $\theta(t)=0$ for $t<0$, and 1 for $t>0$. On using the wave equation (1), the property

$$
\partial_{t} \theta(t)=\delta(t)
$$

and the quantization conditions (15), it is easy to verify that

$$
\begin{aligned}
{\left[\varepsilon(\mathbf{x}) \partial_{t}^{2}-\nabla^{2}\right] G\left(\mathbf{x}, t ; \mathbf{x}^{\prime}, t^{\prime}\right)=} & i \delta\left(t-t^{\prime}\right) \\
& \times\left\langle 0\left|\left[\varepsilon(\mathbf{x}) \dot{\phi}(x), \phi\left(x^{\prime}\right)\right]\right| 0\right\rangle \\
= & \delta^{4}\left(x-x^{\prime}\right)
\end{aligned}
$$

where $\delta^{4}\left(x-x^{\prime}\right)=\delta\left(t-t^{\prime}\right) \delta\left(\mathbf{x}-\mathbf{x}^{\prime}\right)$. The propagator function $G$ defined by Eq. (16) is, therefore, the Green's function of the wave equation (1). We note that by its definition (16), the Green's function is symmetric:

$$
G\left(x, x^{\prime}\right)=G\left(x^{\prime}, x\right),
$$

where we have opted for the four-dimensional notation: $G\left(x, x^{\prime}\right)=G\left(\mathbf{x}, t ; \mathbf{x}^{\prime}, t^{\prime}\right)$, etc.

To each conservation law there is a corresponding Ward identity, which relates a vertex function to the Green's function. The original Ward identity [7] and its generalization, the Ward-Takahashi identity [6], are mathematical statements of electric charge conservation. In classical wave systems like the scalar wave system, there is no charge involved; the original Ward-Takahashi identity [1] obviously does not apply. In cases of classical waves in static inhomogeneous media, it is the energy conservation and the corre- sponding Ward-Takahashi identity that are relevant. These two types of Ward-Takahashi identities are naturally different in structure.

We follow the well-established procedure, due to Takahashi [6], to derive the Ward-Takahashi identity for the energy conservation. For this purpose, we consider the threepoint function $\left\langle 0\left|T\left\{T^{\mu 0}(x) \phi(y) \phi(z)\right\}\right| 0\right\rangle$, which has six possible orderings of the three times associated with $x, y$, and $z$. As a consequence of the energy conservation relation (13), we obtain

$$
\begin{aligned}
\partial_{\mu}^{x} T\left\{T^{\mu 0}(x) \phi(y) \phi(z)\right\}= & \delta\left(x^{0}-y^{0}\right) T\left\{\left[T^{00}(x), \phi(y)\right] \phi(z)\right\} \\
& +\delta\left(x^{0}-z^{0}\right) T\{\phi(y) \\
& \left.\times\left[T^{00}(x), \phi(z)\right]\right\}
\end{aligned}
$$

The equal-time commutators in the above equation can be easily evaluated by using the quantization conditions (15), to yield

$$
\delta\left(x^{0}-y^{0}\right)\left[T^{00}(x), \phi(y)\right]=\frac{1}{i} \delta^{4}(x-y) \dot{\phi}(y), \quad \text { etc. }
$$

Because of

$$
\begin{aligned}
T\{\dot{\phi}(y) \phi(z)\} & =\frac{\partial}{\partial t_{y}} T\{\phi(y) \phi(z)\}-\delta\left(y^{0}-z^{0}\right)[\phi(y), \phi(z)] \\
& =\frac{\partial}{\partial t_{y}} T\{\phi(y) \phi(z)\}
\end{aligned}
$$

from Eq. (21) we obtain the relation

$$
\begin{aligned}
i \partial_{\mu}^{x} T\left\{T^{\mu 0}(x) \phi(y) \phi(z)\right\}= & \delta^{4}(x-y) \frac{\partial}{\partial t_{y}} T\{\phi(y) \phi(z)\} \\
& +\delta^{4}(x-z) \frac{\partial}{\partial t_{z}} T\{\phi(y) \phi(z)\}
\end{aligned}
$$

which, upon taking the "vacuum', expectation value, yields

$$
\begin{aligned}
\partial_{\mu}^{x}\left\langle 0\left|T\left\{T^{\mu 0}(x) \phi(y) \phi(z)\right\}\right| 0\right\rangle= & -\delta^{4}(x-y) \frac{\partial}{\partial t_{x}} G(x, z) \\
& -\delta^{4}(x-z) \frac{\partial}{\partial t_{x}} G(y, x) .
\end{aligned}
$$

This equation provides the basis for the derivation in the next section of the Ward-Takahashi identity for energy conservation in disordered media.

\section{DIFFUSIVE TRANSPORT OF SCALAR WAVES IN DISORDERED MEDIA}

Theoretical description of diffusive behaviors of classical waves in disordered media is of great interest. For example, the analog of the electronic Anderson localization [13] in classical wave systems [14] is directly related to the diffusive nature of wave transport as a result of multiple (elastic and 
inelastic) scatterings. While in the case of an electronic system the conservation law of electric charge underlies the diffusion of electrons, it is the conservation law of energy that underlies the diffusive transport of classical waves. It is, therefore, of importance to have a clear understanding of the implications of the energy conservation in a diffusive environment.

In disordered media, the diffusive transport of scalar waves takes place as a result of random multiple elastic scatterings of waves by the disordered scatterers, phenomenologically represented by the parametric function $\varepsilon(\vec{x})$. The disordered nature of the medium is accounted for by considering a statistical ensemble of medium configurations [15]. The diffusive wave transport behavior, which is stochastic in nature, is obtained by averaging the effects of multiple scatterings over different configurations of the ensemble. This statistical treatment we refer to as ensemble averaging or configuration averaging [9].

The "vacuum" expectation value $\left\langle 0\left|T\left\{T^{\mu 0}(x) \phi(y) \phi(z)\right\}\right| 0\right\rangle$ in the preceding section, in terms of a diagram, is two waves emerging from the energy vertex at $x$, one propagating to $y$, and the other to $z$, undergoing multiple scatterings with the scatterers of the medium along the way. When configuration averaging is applied to it, the disordered medium mediates an effective interaction, statistical in nature, in the wave system [15]. The net effect is the introduction of an extended structure to the energy vertex and the "averaging" of the wave propagation functions. This chain of considerations dictates the following definition of the energy-vertex function $\Gamma_{\mu}(\eta|x| \zeta)$ by

$$
\begin{aligned}
\langle 0| & T\left\{T_{\mu 0}(x) \phi(y) \phi(z)\right\}|0\rangle^{\mathrm{av}} \\
= & \int d^{4} \eta d^{4} \varsigma-\frac{1}{i} G^{(e)}(y-\eta) \Gamma_{\mu}(\eta|x| \mathrm{s}) \frac{1}{i} G^{(e)}(\mathrm{s}-z),
\end{aligned}
$$

where the left-hand side is the configurationally averaged "vacuum" expectation value, and $G^{(e)}$ the averaged propagation function. Use has also been made of the fact that the averaged propagation function depends only on the difference of the two coordinates.

Application of configuration averaging to both sides of Eq. (24) yields the following equation:

$$
\begin{aligned}
\partial_{x}^{\mu} & \int d^{4} \eta d^{4} \varsigma G^{(e)}(y-\eta) \Gamma_{\mu}(\eta|x| \mathrm{s}) G^{(e)}(\mathrm{s}-z) \\
& =\delta^{4}(x-y) \frac{\partial}{\partial t_{x}} G^{(e)}(x-z)+\delta^{4}(x-z) \frac{\partial}{\partial t_{x}} G^{(e)}(y-x) .
\end{aligned}
$$

Making use of the inverse of the Green's function $G^{(e)-1}\left(z-\varsigma^{\prime}\right)$, defined by

$$
\int d^{4} z G^{(e)}(\mathrm{s}-z) G^{(e)-1}\left(z-\varsigma^{\prime}\right)=\delta^{4}\left(\varsigma-\varsigma^{\prime}\right), \quad \text { etc. }
$$

we obtain from Eq. (26) the Ward-Takahashi identity for energy conservation:

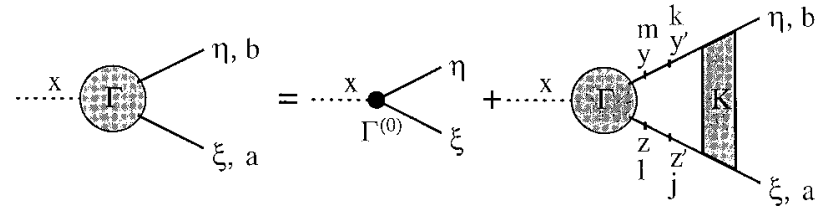

FIG. 1. Generic diagram showing the schematic content of Eqs. (30), (63), and (87).

$$
\begin{aligned}
\partial_{x}^{\mu} \Gamma_{\mu}(\eta|x| \varsigma)= & G^{(e)-1}(\eta-x) \frac{\partial}{\partial t_{x}} \delta^{4}(x-\varsigma) \\
& +G^{(e)-1}(x-\varsigma) \frac{\partial}{\partial t_{x}} \delta^{4}(\eta-x) .
\end{aligned}
$$

This is to be contrasted with the original Ward-Takahashi identity $[6,7,16]$ for charge conservation, which is of the form

$$
\begin{aligned}
\partial_{x}^{\mu} \Gamma_{\mu}^{(\text {charge })}(\eta|x| \varsigma)= & \widetilde{G}^{-1}(\eta, x) \frac{1}{i} \delta^{4}(x-\varsigma) \\
& -\widetilde{G}^{-1}(x, \varsigma) \frac{1}{i} \delta^{4}(\eta-x)
\end{aligned}
$$

where $\Gamma_{\mu}^{(\text {charge })}$ is the charge vertex function, and $\widetilde{G}$ is the electron Green's function. While Eq. (29) describes the flow of charge, Eq. (28) describes the flow of energy, signified by the unmistakable signature of the linear time derivative.

It is more useful to derive an alternative version of the Ward identity that relates various scattering effects, without the involvement of the vertex function $\Gamma_{\mu}$. This can be achieved with the help of an integral equation that relates the energy vertex function $\Gamma_{\mu}$ to the wave-wave, or two-particle, scattering kernel $K$. Considerations similar to those that are used to derive the Bethe-Salpeter equation [15] lead to the integral equation

$$
\begin{aligned}
\Gamma_{\mu}(\eta|x| \varsigma)= & \Gamma_{\mu}^{(0)}(\eta|x| \varsigma)-\int d^{4} y d^{4} y^{\prime} d^{4} z d^{4} z^{\prime} G^{(e)} \\
& \times\left(z^{\prime}-z\right) \Gamma_{\mu}(z|x| y) G^{(e)}\left(y-y^{\prime}\right) K\left(\eta \varsigma ; y^{\prime} z^{\prime}\right),
\end{aligned}
$$

which, we note, has a structure similar to a corresponding integral equation in quantum electrodynamics [17]. Here, $\Gamma_{\mu}^{(0)}$ denotes the vertex function for a homogeneous reference medium, and $K\left(\eta s ; y^{\prime} z^{\prime}\right)$ the irreducible wave-wave scattering kernel, usually referred to as the Bethe-Salpeter kernel.

The structure of the integral equation (30) is schematically shown in Fig. 1. Heuristically, the picture is as follows. At the vertex emerge two waves, which undergo multiple scatterings with the medium as well as (effective) collisions with each other as they propagate. The integral equation is an economic representation of those multiple scatterings in terms of the Green's functions, which include all scattering effects with the medium. The irreducible "two-particle" kernel, $K\left(\eta \zeta ; y^{\prime} z^{\prime}\right)$, summarizes the (effective) wave-wave scatterings [17] from the two input channels $(\eta ; \zeta)$ to the two output channels $\left(y^{\prime} ; z^{\prime}\right)$. 
By differentiating Eq. (30) and using Eq. (26), we obtain

$$
\begin{aligned}
\partial_{x}^{\mu} \Gamma_{\mu}(\eta|x| \zeta)-\partial_{x}^{\mu} \Gamma_{\mu}^{(0)}(\eta|x| \zeta) \\
=-\int d^{4} y d^{4} z\left[\delta^{4}(x-z) \frac{\partial}{\partial t_{x}} G^{(e)}(x-y)\right. \\
\left.\quad+\delta^{4}(x-y) \frac{\partial}{\partial t_{x}} G^{(e)}(z-x)\right] K(\eta \zeta ; y z) .
\end{aligned}
$$

The use of Eq. (28) yields

$$
\begin{aligned}
& {\left[G^{(e)-1}(\eta-x)-G^{(0)-1}(\eta-x)\right] \frac{\partial}{\partial t_{x}} \delta^{4}(x-\zeta)} \\
& \quad+\left[G^{(e)-1}(x-\zeta)-G^{(0)-1}(x-\zeta)\right] \frac{\partial}{\partial t_{x}} \delta^{4}(\eta-x) \\
& =-\int d^{4} y d^{4} z\left[\delta^{4}(x-z) \frac{\partial}{\partial t_{x}} G^{(e)}(x-y)\right. \\
& \left.\quad+\delta^{4}(x-y) \frac{\partial}{\partial t_{x}} G^{(e)}(z-x)\right] K^{(e)}(\eta \zeta ; y z),
\end{aligned}
$$

where $G^{(0)}$ is the Green's function for the homogeneous reference medium. Due to configurational averaging over the coordinates of the scatterers, only three of the four coordinates in $K(\eta \zeta ; y z)$ are independent. We choose $(\eta-y),(\zeta$ $-z)$, and $(y-z)$ to be the three independent coordinates. Furthermore, since the two input channels are mediated by an effective static potential which is time independent, implied by the independence of $\varepsilon(\mathbf{x})$ on time, $(y-z)$ can therefore be replaced by $(\mathbf{y}-\mathbf{z})$. We shall use the notation

$$
K(\eta \zeta ; y z)=U(\eta-y, \zeta-z, \mathbf{y}-\mathbf{z}) .
$$

By using the inverse of the configurationally averaged Green's function in the form

$$
G^{(e)-1}=G^{(0)-1}-\Sigma,
$$

where $\Sigma$ is the self-energy operator that accounts for all the multiple scatterings, one obtains the multiple-scattering form of the Ward identity, which we shall name the diffusive Ward identity:

$$
\begin{aligned}
& \Sigma(\eta-x) \frac{\partial}{\partial t_{x}} \delta^{4}(x-\zeta)+\Sigma(x-\zeta) \frac{\partial}{\partial t_{x}} \delta^{4}(\eta-x) \\
& =\int d^{4} y d^{4} z\left[\delta^{4}(x-z) \frac{\partial}{\partial t_{x}} G(x-y)+\delta^{4}(x-y) \frac{\partial}{\partial t_{x}}\right. \\
& \quad \times G(z-x)] U(\eta-y, \zeta-z, \mathbf{y}-\mathbf{z}) .
\end{aligned}
$$

In momentum space, the diffusive Ward identity is of the form

$$
\begin{aligned}
q_{0} \Sigma(q+p)-\left(q_{0}+p_{0}\right) \Sigma(q) \\
=\int \frac{d^{3} \boldsymbol{\omega}}{(2 \pi)^{3}}\left[q_{0} G^{(e)}(q+\boldsymbol{\omega})-\left(q_{0}+p_{0}\right)\right. \\
\left.\quad \times G^{(e)}(q+p+\boldsymbol{\omega})\right] U(-q, q+p, \boldsymbol{\omega}),
\end{aligned}
$$

where

$$
\Sigma(x)=\int \frac{d^{4} q}{(2 \pi)^{4}} e^{i q \cdot x} \Sigma(q), \quad \text { etc. }
$$

We note that the energy coefficients $q_{0}$ and $\left(q_{0}+p_{0}\right)$ in Eq. (36) reflect the time derivatives in Eq. (35), which can be traced to the time derivatives in the basic Ward-Takahashi identities (26) or (28). We remind ourselves that the linear time derivative is a signature of energy flow. In the more conventional form, the diffusive Ward identity is expressed as

$$
\begin{aligned}
\int & \frac{d^{3} \boldsymbol{\omega}}{(2 \pi)^{3}}\left[G^{(e)}(q+\boldsymbol{\omega})-G^{(e)}(q+p+\boldsymbol{\omega})\right] \\
& \times U(-q, q+p, \boldsymbol{\omega}) \\
= & \Sigma(q+p)-\Sigma(q)-E(q, p),
\end{aligned}
$$

with

$$
\begin{aligned}
E(q, p)= & \frac{p_{0}}{q_{0}}\left[\Sigma(q)-\int \frac{d^{3} \boldsymbol{\omega}}{(2 \pi)^{3}} G^{(e)}(q+p+\boldsymbol{\omega})\right. \\
& \times U(-q, q+p, \boldsymbol{\omega})] .
\end{aligned}
$$

The diffusive Ward identity is crucial for the derivation of wave diffusive transport behavior on the basis of the BetheSalpeter equation, the basic reason being that it is the energy that is being diffusively transported. As such, it inevitably affects the wave diffusion constant, which is generally recognized to be the product of the transport velocity and the transport mean free path. In this context, we point out that the term $E(q, p)$ in Eq. (38) has indeed been recognized to affect the energy transport velocity that appears in the diffusion constant, especially near resonant scatterings [10]. On the other hand, as is clear from the derivation of the diffusive Ward identity (38), the origin of the $E(q, p)$ term can be traced to the presence of the time derivatives in the original form of the Ward-Takahashi identities (26) or (28).

We note that our result (36) or (38) differs from the result of Ref. [4]. The difference will be discussed in a later section when we discuss the case of electromagnetic waves.

\section{ALTERNATIVE VERSION OF THE SCALAR WAVE}

Another version of the scalar wave equation is

$$
\left[\partial_{t}^{2}-\nabla \cdot \frac{1}{\varepsilon(\mathbf{x})} \nabla\right] \phi(\mathbf{x}, t)=0 .
$$

The Lagrangian density corresponding to this equation is

$$
L^{(\mathrm{S} 2)}=\frac{1}{2}\left[\partial_{t} \phi \partial_{t} \phi-\frac{1}{\varepsilon(\mathbf{x})} \boldsymbol{\nabla} \phi \cdot \nabla \phi\right] .
$$

In this case, the energy-momentum densities, according to the general formula (10), are

$$
\begin{gathered}
T_{00}^{(\mathrm{S} 2)}=\frac{1}{2}\left(\dot{\phi} \dot{\phi}+\frac{1}{\varepsilon} \nabla \phi \cdot \nabla \phi\right), \\
T_{k 0}^{(\mathrm{S} 2)}=\frac{1}{\varepsilon} \partial_{k} \phi \partial_{0} \phi,
\end{gathered}
$$


which again satisfies the conservation equation

$$
\partial^{\mu} T_{\mu 0}^{(\mathrm{S} 2)}=0 .
$$

The same quantization conditions (15) are imposed on the field $\phi$ and its conjugate, which is

$$
\pi^{(\mathrm{S} 2)}(\mathbf{x}, t)=\dot{\phi}(\mathbf{x}, t) .
$$

It can be easily verified that the propagator function, defined by

$$
G\left(x, x^{\prime}\right)=i\left\langle 0\left|T\left\{\phi(x) \phi\left(x^{\prime}\right)\right\}\right| 0\right\rangle,
$$

satisfies

$$
\left[\partial_{t}^{2}-\nabla \cdot \frac{1}{\varepsilon(\mathbf{x})} \boldsymbol{\nabla}\right] G\left(x, x^{\prime}\right)=\delta^{4}\left(x-x^{\prime}\right),
$$

and is the Green's function for the scalar wave equation (2).

We can proceed in complete parallel to the previous case to derive the basic Ward-Takahashi identities (26) or (28) and the diffusive Ward identities (36) or (38). The forms of the Ward identities are exactly the same as the previous case. Only the precise contents of the Green's functions are different in the two cases.

\section{ELASTIC WAVE CASE}

The case of the elastic wave has been considered in detail elsewhere [11]. For completeness, we present a brief account here. The elastic wave equation is

$$
\rho \ddot{s}_{j}-\partial_{i}\left[\lambda(\boldsymbol{\nabla} \cdot \mathbf{s}) \delta_{i j}+\mu\left(\partial_{i} s_{j}+\partial_{j} s_{i}\right)\right]=0,
$$

or,

$$
O_{i j} s_{j}=0 \text {, }
$$

where

$$
O_{i j}=\rho\left(\partial_{i}\right)^{2} \delta_{i j}-\partial_{i}\left(\lambda \partial_{j}\right)-\partial_{j}\left(\mu \partial_{i}\right)-\delta_{i j} \nabla \cdot(\mu \nabla) .
$$

The Lagrangian density for the elastic wave equation is given by

$$
L^{(\mathrm{EL})}=\frac{1}{2}\left[\rho(\dot{\mathbf{s}})^{2}-\lambda\left(\operatorname{Tr} \varepsilon_{i j}\right)^{2}-2 \mu \varepsilon_{i j} \varepsilon_{j i}\right],
$$

with

$$
\varepsilon_{i j}=\frac{1}{2}\left(\partial_{i} s_{j}+\partial_{j} s_{i}\right) .
$$

The formula for the energy-momentum density is

$$
T_{\mu \nu}^{(\mathrm{EL})}=\sum_{i=1}^{3} \frac{\partial L^{(\mathrm{EL})}}{\partial\left(\partial^{\mu} s_{i}\right)} \frac{\partial s_{i}}{\partial x^{\nu}}-g_{\mu \nu} L^{(\mathrm{EL})},
$$

according to which,

$$
T_{00}^{(\mathrm{EL})}=\frac{1}{2}\left[\rho(\dot{\mathbf{s}})^{2}+\lambda\left(\operatorname{Tr} \varepsilon_{i j}\right)^{2}+2 \mu \varepsilon_{i j} \varepsilon_{j i}\right]
$$

and

$$
T_{k 0}^{(\mathrm{EL})}=\lambda(\boldsymbol{\nabla} \cdot \mathbf{s}) \dot{s}_{k}+\mu \dot{s}_{j}\left(\partial_{j} s_{k}+\partial_{k} s_{j}\right) .
$$

The energy conservation equation may be verified to be

$$
\partial^{\mu} T_{\mu 0}^{(\mathrm{EL})}=0
$$

The system is quantized by imposing commutation relations on the fields $s_{i}$ and their conjugates given by

$$
\pi_{i}=\frac{\partial L^{(\mathrm{EL})}}{\partial s_{i}}=\rho \dot{s}_{i}
$$

Quantization conditions are

$$
\left[s_{i}(\mathbf{x}, t), \pi_{j}(\mathbf{y}, t)\right]=i \delta_{i j} \delta(\mathbf{x}-\mathbf{y})
$$

and

$$
\begin{gathered}
{\left[s_{i}(\mathbf{x}, t), s_{j}(\mathbf{y}, t)\right]=0,} \\
{\left[\pi_{i}(\mathbf{x}, t), \pi_{j}(\mathbf{y}, t)\right]=0 .}
\end{gathered}
$$

We note that while $s_{i}$ are quantum operators, $\rho$ is a numerical function.

The propagator function $G_{j k}\left(x, x^{\prime}\right)$ is defined by

$$
G_{j k}\left(x, x^{\prime}\right)=i\left\langle 0\left|T\left\{s_{j}(x) s_{k}\left(x^{\prime}\right)\right\}\right| 0\right\rangle .
$$

We note that, by definition,

$$
G_{j k}\left(x, x^{\prime}\right)=G_{k j}\left(x^{\prime}, x\right) .
$$

On account of the elastic wave equation (3) or (45) and the commutation relations (52) and (53), one can straightforwardly prove, as in the scalar wave case, that the propagator function defined in Eq. (54) is the Green's function for the elastic wave equation, and satisfies

$$
O_{i j} G_{j k}\left(x, x^{\prime}\right)=\delta_{i k} \delta^{4}\left(x-x^{\prime}\right)
$$

As in the case of the scalar waves, we consider the "vacuum" expectation value $\left\langle 0\left|T\left\{T_{\mu 0}^{(\mathrm{EL})}(x) s_{1}(y) s_{m}(z)\right\}\right| 0\right\rangle$. We can easily verify that

$$
\begin{aligned}
& \partial_{x}^{\mu} T\left\{T_{\mu 0}^{(\mathrm{EL})}(x) s_{1}(y) s_{m}(z)\right\} \\
&= \delta\left(x^{0}-y^{0}\right) T\left\{\left[T_{00}^{(\mathrm{EL})}(x), s_{1}(y)\right] s_{m}(z)\right\} \\
&+\delta\left(x^{0}-z^{0}\right) T\left\{s_{1}(y)\left[T_{00}^{(\mathrm{EL})}(x), s_{m}(z)\right]\right\} \\
&= \frac{1}{i} \delta^{4}(x-y) T\left\{\dot{s}_{1}(y) s_{m}(z)\right\} \\
&+\frac{1}{i} \delta^{4}(x-z) T\left\{s_{1}(y) \dot{s}_{m}(z)\right\} \\
&= \frac{1}{i} \delta^{4}(x-y) \frac{\partial}{\partial t_{y}} T\left\{s_{1}(y) s_{m}(z)\right\} \\
&+\frac{1}{i} \delta^{4}(x-z) \frac{\partial}{\partial t_{z}} T\left\{s_{1}(y) s_{m}(z)\right\},
\end{aligned}
$$

from which we obtain 


$$
\begin{aligned}
\partial_{x}^{\mu}\langle 0| & T\left\{T_{\mu 0}^{(\mathrm{EL})}(x) s_{1}(y) s_{m}(z)\right\}|0\rangle \\
= & -\delta^{4}(x-y) \frac{\partial}{\partial t_{x}} G_{l m}(x, z)-\delta^{4}(x-z) \frac{\partial}{\partial t_{x}} G_{l m}(y, x) .
\end{aligned}
$$

The energy irreducible vertex function $\Gamma_{\mu}^{j k}(\eta|x| \zeta)$, defined by

$$
\begin{aligned}
&\langle 0| T\left\{T_{\mu 0}^{(\mathrm{EL})}(x) s_{1}(y) s_{m}(z)\right\}|0\rangle^{\mathrm{av}} \\
& \quad=\int d^{4} \eta d^{4} \zeta \frac{1}{i} G_{l j}^{(e)}(y-\eta) \Gamma_{\mu}^{j k}(\eta|x| \zeta) \frac{1}{i} G_{k m}^{(e)}(\zeta-z),
\end{aligned}
$$

satisfies

$$
\begin{aligned}
\partial_{x}^{\mu} \int & d^{4} \eta d^{4} \zeta G_{l j}^{(e)}(y-\eta) \Gamma_{\mu}^{j k}(\eta|x| \zeta) G_{k m}^{(e)}(\zeta-z) \\
& =\delta^{4}(x-y) \frac{\partial}{\partial t_{x}} G_{l m}^{(e)}(x-z)+\delta^{4}(x-z) \frac{\partial}{\partial t_{x}} G_{l m}^{(e)}(y-x) .
\end{aligned}
$$

Making use of the inverse of the Green's function $G_{i l}^{(e)-1}\left(\eta^{\prime}, y\right)$, defined by

$$
\int d^{4} y G_{i l}^{(e)-1}\left(\eta^{\prime}-y\right) G_{l j}^{(e)}(y-\eta)=\delta_{i j} \delta^{4}\left(\eta^{\prime}-\eta\right), \quad \text { etc. }
$$

we obtain the energy Ward-Takahashi identity for the elastic wave in its basic form:

$$
\begin{aligned}
\partial_{x}^{\mu} \Gamma_{\mu}^{i j}(\eta|x| \zeta)= & G_{i j}^{(e)-1}(\eta-x) \frac{\partial}{\partial t_{x}} \delta^{4}(x-\zeta) \\
& +G_{i j}^{(e)-1}(x-\zeta) \frac{\partial}{\partial t_{x}} \delta^{4}(\eta-x) .
\end{aligned}
$$

Except for the vector indices in the definitions of the vertex function and the Green's function, the structure of this identity is of the same form as the corresponding identity (28) for the scalar wave. Again, there is the appearance of the linear time derivative, which is a signature for energy flow.

To derive the diffusive Ward identity for the elastic wave, we make use of an integral equation for the vertex function similar to the integral equation (30) for the scalar wave case. It is

$$
\begin{aligned}
\Gamma_{\mu}^{a b}(\eta|x| \zeta) \\
=\Gamma_{\mu}^{(0) a b}(\eta|x| \zeta)-\int d^{4} y d^{4} y^{\prime} d^{4} z d^{4} z^{\prime} G_{j l}^{(e)}\left(z-z^{\prime}\right) \\
\quad \times \Gamma_{\mu}^{i m}\left(z^{\prime}|x| y^{\prime}\right) G_{m i}^{(e)}\left(y^{\prime}-y\right) K_{a b ; i j}(\eta \zeta ; y z),
\end{aligned}
$$

and is understood to hold for the configurationally averaged quantities. Here, $K_{a b ; i j}(\eta \zeta ; y z)$ is the "two-particle" BetheSalpeter kernel that also appears in the Bethe-Salpeter equation. The use of Eq. (62), in terms of the configurationally averaged quantities, gives

$$
\begin{aligned}
& {\left[G_{a b}^{(e)-1}(\eta-x)-G_{a b}^{(0)-1}(\eta-x)\right] \frac{\partial}{\partial t_{x}} \delta^{4}(x-\zeta)} \\
& \quad+\left[G_{a b}^{(e)-1}(x-\zeta)-G_{a b}^{(0)-1}(x-\zeta)\right] \frac{\partial}{\partial t_{x}} \delta^{4}(\eta-x) \\
& \quad=-\int d^{4} y d^{4} z\left[\delta^{4}(x-z) \frac{\partial}{\partial t_{x}} G_{j i}^{(e)}(x-y)\right. \\
& \left.\quad+\delta^{4}(x-y) \frac{\partial}{\partial t_{x}} G_{j i}^{(e)}(z-x)\right] U_{a b ; i j}(\eta-y, \zeta-z, \mathbf{y}-\mathbf{z})
\end{aligned}
$$

where $G_{a b}^{(0)}$ is the Green's function in the homogeneous reference medium, and

$$
K_{a b ; i j}(\eta \zeta ; y z)=U_{a b ; i j}(\eta-y, \zeta-z, \mathbf{y}-\mathbf{z})
$$

Again, the static property of the medium has been made use of, namely, the parametric functions $\rho(\mathbf{x})$ and $\mu(\mathbf{x})$, are independent of time. In terms of the "self-energy" $\Sigma_{a b}$, defined by

$$
G_{a b}^{(e)-1}=G_{a b}^{(0)-1}-\Sigma_{a b},
$$

from Eq. (64) one obtains the energy diffusive Ward identity for elastic waves:

$$
\begin{aligned}
\Sigma_{a b}( & \eta-x) \frac{\partial}{\partial t_{x}} \delta^{4}(x-\zeta)+\Sigma_{a b}(x-\zeta) \frac{\partial}{\partial t_{x}} \delta^{4}(\eta-x) \\
= & \int d^{4} y d^{4} z\left[\delta^{4}(x-z) \frac{\partial}{\partial t_{x}} G_{j i}^{(e)}(x-y)\right. \\
& \left.+\delta^{4}(x-y) \frac{\partial}{\partial t_{x}} G_{j i}^{(e)}(z-x)\right] \\
& \times U_{a b ; i j}(\eta-y, \zeta-z, \mathbf{y}-\mathbf{z}),
\end{aligned}
$$

or, in terms of the momentum-space representation,

$$
\begin{aligned}
q_{0} \Sigma_{a b}(q+p)-\left(q_{0}+p_{0}\right) \Sigma_{a b}(q) \\
=\int \frac{d^{3} \boldsymbol{\omega}}{(2 \pi)^{3}}\left[q_{0} G_{j i}^{(e)}(q+\boldsymbol{\omega})\right. \\
\left.\quad-\left(q_{0}+p_{0}\right) G_{j i}^{(e)}(q+p+\boldsymbol{\omega})\right] U_{a b ; j i}(-q, q+p, \boldsymbol{\omega}),
\end{aligned}
$$

where

$$
\Sigma_{a b}(x)=\int \frac{d^{4} q}{(2 \pi)^{4}} e^{i q \cdot x} \Sigma_{a b}(q), \quad \text { etc. }
$$

Another form is 


$$
\begin{aligned}
\int & \frac{d^{3} \boldsymbol{\omega}}{(2 \pi)^{3}}\left[G_{j i}^{(e)}(q+\boldsymbol{\omega})-G_{j i}^{(e)}(1+p+\boldsymbol{\omega})\right] U_{a b ; i j} \\
& \times(-q, q+p, \boldsymbol{\omega})=\Sigma_{a b}(q+p)-\Sigma_{a b}(q)-E_{a b}(q, p),
\end{aligned}
$$

with

$$
\begin{aligned}
E_{a b}(q, p)= & \frac{p_{0}}{q_{0}}\left[\Sigma_{a b}(q)-\int \frac{d^{3} \boldsymbol{\omega}}{(2 \pi)^{3}} G_{j i}^{(e)}(q+p+\boldsymbol{\omega}) U_{a b ; i j}\right. \\
& \times(-q, q+p, \boldsymbol{\omega})] .
\end{aligned}
$$

\section{ELECTROMAGNETIC WAVE CASE}

The case of the electromagnetic wave is similar to but much more complicated than the case of the elastic wave. The tricky complication here is caused by the well-known gauge freedom of the electromagnetic field. Fortunately, there exists a wealth of accumulated knowledge on how to deal with and even to exploit the gauge freedom in gauge theories, of which the quantum electrodynamics is the simplest. One lesson learned is that one can judiciously choose a particular gauge for a particular purpose, knowing that the final result is independent of the gauge chosen. In our present case of electromagnetic waves, the field equations are the Maxwell equations

$$
\begin{gathered}
\partial_{k} H^{k 0}=0, \\
\partial_{0} H^{01}+\partial_{k} H^{k l}=0,
\end{gathered}
$$

or

$$
\partial_{\mu} H^{\mu \nu}=0
$$

where

$$
\begin{gathered}
H^{0 k}=\varepsilon F^{0 k}, \\
H^{k l}=\mu F^{k l} .
\end{gathered}
$$

In terms of the four-potential $A_{\mu}$, the field strength tensor is

$$
F_{\mu \nu}=\partial_{\mu} A_{\nu}-\partial_{\nu} A_{\mu} .
$$

It is clear that Eq. (9) is invariant under gauge transformations. Thus, in material media (as opposed to vacuum), we still have a gauge invariant theory. In our considerations to follow it is most convenient to choose a gauge such that

$$
A_{0}=0,
$$

This is the so-called temporal gauge [18]. This gauge condition eliminates one of the four apparent degrees of freedom represented by the four-potential $A_{\mu}$. Since the Maxwell system has only two intrinsic degrees of freedom, we need to impose another condition in addition to Eq. (71). It is known [18] that this additional condition is a constraint on the physical states based on Eq. (8a):

$$
\partial_{k} H^{k 0}|\psi\rangle=0 .
$$

This is the so-called Gauss-law constraint. In this gauge, the equation of motion (8b) is of a form that involves only the spatial components of the vector potential:

$$
\widetilde{O}^{i}{ }_{j} A^{j}=0,
$$

where

$$
\begin{aligned}
\widetilde{O}^{i}{ }_{j}= & \varepsilon\left(\partial_{t}\right)^{2} \delta_{j}^{i}+\left(\partial_{k} \mu\right)\left(\delta_{j}^{i} \partial^{k}-\delta^{k}{ }_{j} \partial^{i}\right) \\
& +\mu\left(\delta^{i}{ }_{j} \partial_{k} \partial^{k}-\delta^{k}{ }_{j} \partial_{k} \partial^{i}\right) .
\end{aligned}
$$

The Lagrangian density is

$$
L^{(\mathrm{EM})}=-\frac{1}{4} H^{\mu \nu} F_{\mu \nu} .
$$

The canonical conjugate to $A_{i}$ is

$$
\pi^{i}=\frac{\partial L^{(\mathrm{EM})}}{\partial\left(\partial^{0} A_{i}\right)}=H^{i 0}=\varepsilon(\mathbf{x})\left(\partial^{i} A^{0}-\partial^{0} A^{i}\right) .
$$

Under the weak constraint condition (72), the quantization conditions are [19]

$$
\left[A_{i}(\mathbf{x}, t), \pi^{j}(\mathbf{y}, t)\right]=i \delta_{i}^{j} \delta^{3}(\mathbf{x}-\mathbf{y}),
$$

and

$$
\begin{aligned}
& {\left[A^{i}(\mathbf{x}, t), A^{j}(\mathbf{y}, t)\right]=0,} \\
& {\left[\pi^{i}(\mathbf{x}, t), \pi^{j}(\mathbf{y}, t)\right]=0 .}
\end{aligned}
$$

The energy-momentum tensor is given by

$$
T_{\mu \nu}^{(\mathrm{EM})}=H_{\mu}{ }^{\lambda} F_{\lambda \nu}-g_{\mu \nu} L^{(\mathrm{EM})} .
$$

The energy conservation equation is

$$
\partial^{\mu} T_{\mu 0}^{(\mathrm{EM})}=0 .
$$

For the electromagnetic field, the propagator function $\widetilde{G}^{j k}(x, y)$ is similarly defined by

$$
\widetilde{G}^{j k}(x, y)=i\left\langle 0\left|T\left\{A^{j}(x) A^{k}(y)\right\}\right| 0\right\rangle,
$$

which can be verified to satisfy [19]

$$
\begin{aligned}
\widetilde{O}_{(x)}^{i}{ }_{j} \widetilde{G}^{j k}(x, y) & =i \delta\left(x^{0}-y^{0}\right)\left\langle 0\left|\left[\varepsilon(\mathbf{x}) \dot{A}^{i}(x), A^{k}(y)\right]\right| 0\right\rangle \\
& =\delta^{i k} \delta^{4}(x-y)
\end{aligned}
$$

and is, therefore, the Green's function for the wave equation (73). We note that in the derivation of Eq. (81) the temporal gauge condition (71) played a crucially important role; it is in this gauge only that the propagator function defined by Eq. (80) is the Green's function satisfying Eq. (81).

Again, we consider the "vacuum" expectation value of $T\left\{T_{\mu 0}^{(\mathrm{EM})}(x) A_{1}(y) A_{m}(z)\right\}$, for which we can similarly prove, on using the commutation relations (76) and (77) as well as the temporal gauge condition (71), that 


$$
\begin{aligned}
\partial_{x}^{\mu}\langle 0| & T\left\{T_{\mu 0}^{(\mathrm{EM})}(x) A_{1}(y) A_{m}(z)\right\}|0\rangle \\
= & \frac{1}{i} \delta^{4}(x-y)\left\langle 0\left|T\left\{F_{01}(x) A_{m}(z)\right\}\right| 0\right\rangle \\
& +\frac{1}{i} \delta^{4}(x-z)\left\langle 0\left|T\left\{F_{0 m}(x) A_{1}(y)\right\}\right| 0\right\rangle \\
= & \frac{1}{i} \delta^{4}(x-y)\langle 0| T\left\{\dot{A}_{1}(x) A_{m}(z)|0\rangle\right. \\
& +\frac{1}{i} \delta^{4}(x-z)\left\langle 0\left|T\left\{\dot{A}_{m}(x) A_{1}(y)\right\}\right| 0\right\rangle \\
= & -\delta^{4}(x-y) \frac{\partial}{\partial t_{x}} \widetilde{G}_{l m}(x, z) \\
& -\delta^{4}(x-z) \frac{\partial}{\partial t_{x}} \widetilde{G}_{m l}(x, y) .
\end{aligned}
$$

Again, the temporal gauge condition (71) is crucial in the above derivation. The irreducible energy vertex function $\widetilde{\Gamma}_{\mu}^{j k}(\xi|x| \eta)$ is defined by

$$
\begin{aligned}
&\langle 0| T\left\{T_{\mu 0}^{(\mathrm{EM})}(x) A_{1}(y) A_{m}(z)\right\}|0\rangle^{\mathrm{av}} \\
& \quad=\int d^{4} \eta d^{4} \zeta \frac{1}{i} \widetilde{G}_{l j}^{(e)}(y-\eta) \widetilde{\Gamma}_{\mu}^{j k}(\eta|x| \zeta) \frac{1}{i} \widetilde{G}_{k m}^{(e)}(\zeta-z) .
\end{aligned}
$$

It then follows from Eq. (82) that

$$
\begin{aligned}
\partial_{x}^{\mu} \int & d^{4} \eta d^{4} \zeta G_{l j}^{(e)}(y-\eta) \Gamma_{\mu}^{j k}(\eta|x| \zeta) \widetilde{G}_{k m}^{(e)}(\zeta-z) \\
= & \delta^{4}(x-y) \frac{\partial}{\partial t_{x}} \widetilde{G}_{l m}^{(e)}(x-z) \\
& +\delta^{4}(x-z) \frac{\partial}{\partial t_{x}} \widetilde{G}_{l m}^{(e)}(y-x) .
\end{aligned}
$$

Upon using the inverse of the Green's function $\widetilde{\mathrm{G}}_{i l}^{(e)-1}\left(\eta^{\prime}\right.$ $-y$ ), defined by

$$
\int d^{4} y \widetilde{G}_{i l}^{(e)-1}\left(\eta^{\prime}-y\right) \widetilde{G}_{l j}(y, \eta)=\delta_{i j} \delta^{4}\left(\eta^{\prime}-\eta\right), \quad \text { etc. }
$$

we obtain the basic Takahashi-Ward identity corresponding to energy conservation:

$$
\begin{aligned}
\partial_{x}^{\mu} \Gamma_{\mu}^{i j}(\eta|x| \zeta)= & \widetilde{G}_{i j}^{(e)-1}(\eta-x) \frac{\partial}{\partial t_{x}} \delta^{4}(x-\zeta) \\
& +\widetilde{G}_{i j}^{(e)-1}(x-\zeta) \frac{\partial}{\partial t_{x}} \delta^{4}(\eta-x)
\end{aligned}
$$

To apply this basic identity to the diffusive transport of electromagnetic waves, we again make use of the integral equation for the irreducible energy vertex:

$$
\begin{aligned}
\widetilde{\Gamma}_{\mu}^{a b}(\eta|x| \zeta)= & \widetilde{\Gamma}_{\mu}^{(0) a b}(\eta|x| \zeta) \\
& -\int d^{4} y d^{4} y^{\prime} d^{4} z d^{4} z^{\prime} \widetilde{G}_{j l}^{(e)}\left(z^{\prime}-z\right) \widetilde{\Gamma}_{\mu}^{l m}(z|x| y) \\
& \times \widetilde{G}_{m i}^{(e)}\left(y-y^{\prime}\right) \widetilde{K}_{a b ; i j}\left(\eta \zeta ; y^{\prime} z^{\prime}\right)
\end{aligned}
$$

where $\widetilde{K}_{a b ; i j}$ is the Bethe-Salpeter kernel for the electromagnetic wave system. We combine the two equations (84) and (87) to arrive at

$$
\begin{aligned}
\partial_{x}^{\mu} \widetilde{\Gamma}_{\mu}^{a b}(\eta|x| \zeta)-\partial_{x}^{\mu} \widetilde{\Gamma}_{\mu}^{(0) a b}(\eta|x| \zeta) \\
=-\int d^{4} y d^{4} z\left[\delta^{4}(x-z) \frac{\partial}{\partial t_{x}} \widetilde{G}_{j i}^{(e)}(x-y)\right. \\
\left.\quad+\delta^{4}(x-y) \frac{\partial}{\partial t_{x}} \widetilde{G}_{j i}^{(e)}(z-x)\right] \widetilde{K}_{a b ; i j}(\eta \zeta ; y z) .
\end{aligned}
$$

In terms of the "self-energy operator", $\widetilde{\Sigma}_{a b}$, defined by

$$
\widetilde{G}_{a b}^{(e)-1}=\widetilde{G}_{a b}^{(0)-1}-\widetilde{\Sigma}_{a b},
$$

where $\widetilde{G}_{a b}^{(0)}$ is the Green's function for the homogeneous reference medium Eqs. (86) and (88) lead to the following form of the diffusive Ward identity:

$$
\begin{aligned}
\widetilde{\Sigma}_{a b}(\eta-x) \frac{\partial}{\partial t_{x}} \delta^{4}(x-\zeta)+\widetilde{\Sigma}_{a b}(x-\zeta) \frac{\partial}{\partial t_{x}} \delta^{4}(\eta-x) \\
=\int d^{4} y d^{4} z\left[\delta^{4}(x-z) \frac{\partial}{\partial t_{x}} G_{j i}^{(e)}(x-y)+\delta^{4}(x-y)\right. \\
\left.\quad \times \frac{\partial}{\partial t_{x}} \widetilde{G}_{j i}^{(e)}(z-x)\right] \widetilde{U}_{a b ; i j}(\eta-y, \zeta-z, \mathbf{y}-\mathbf{z})
\end{aligned}
$$

In momentum-space representation, Eq. (90) is of the form

$$
\begin{aligned}
q_{0} \widetilde{\Sigma}_{a b}(q+p)-\left(q_{0}+p_{0}\right) \widetilde{\Sigma}_{a b}(q) \\
=\int \frac{d^{3} \boldsymbol{\omega}}{(2 \pi)^{3}}\left[q_{0} G_{j i}^{(e)}(q+\boldsymbol{\omega})\right. \\
\left.\quad-\left(q_{0}+p_{0}\right) G_{j i}^{(e)}(q+p+\boldsymbol{\omega})\right] \widetilde{U}_{a b ; j}(-q, q+p, \boldsymbol{\omega}),
\end{aligned}
$$

where

$$
\widetilde{\Sigma}_{a b}(x)=\int \frac{d q}{(2 \pi)^{4}} e^{i q \cdot x} \widetilde{\Sigma}_{a b}(q), \quad \text { etc. }
$$

or

$$
\begin{aligned}
\int & \frac{d^{3} \omega}{(2 \pi)^{3}}\left[G_{j i}^{(e)}(q+\boldsymbol{\omega})-G_{j i}^{(e)}(q+p+\boldsymbol{\omega})\right] \\
& \times \widetilde{U}_{a b ; i j}(-q, q+p, \boldsymbol{\omega}) \\
= & \widetilde{\Sigma}_{a b}(q+p)-\widetilde{\Sigma}_{a b}(q)-\widetilde{E}_{a b}(q, p),
\end{aligned}
$$

with 


$$
\begin{aligned}
\widetilde{E}_{a b}(q, p)= & \frac{p_{0}}{q_{0}}\left[\widetilde{\Sigma}_{a b}(q)-\int \frac{d^{3} \boldsymbol{\omega}}{(2 \pi)^{3}} G_{j i}^{(e)}(q+p+\boldsymbol{\omega}) \widetilde{U}_{a b ; i j}\right. \\
& \times(-q, q+p, \boldsymbol{\omega})] .
\end{aligned}
$$

We note that the Ward identities in the case of the electromagnetic waves are identical in form to those for the elastic waves. The difference, however, is hidden in the Gauss-law constraint equation (72) imposed on the physical states.

We were brought to the attention of the work of Barabanenkov, Zurk, and Barabanenkov [5], who used a completely different approach to derive a Ward identity for the electromagnetic wave in media with constant magnetic permeability. Their result looks somewhat similar to ours. However, coefficients quadratic in frequencies (energies) permeate throughout their work and appear in their final result, whereas our results, e.g., Eq. (91), show a linear dependence of the coefficients on energy. The origin of the linear dependence, according to our derivation, is clear. It is the linear time derivative in the basic Ward-Takahasi identity (86). As we already emphasized, the linear time derivative is a signature of energy flow. While the version of the Ward identity of Ref. [5] can be interpreted to be consistent with ours in the limit of infinitesimal frequency difference $p_{0}$, it should be noted that our Ward identities, for general $\varepsilon(\mathbf{x})$ and $\mu(\mathbf{x})$, are valid for arbitrary values of the frequency difference $p_{0}$.
The remarks here also apply to the case of scalar waves, where our result is similarly different from the result of Ref. [4].

The system described by the Lagrangian density $L^{(\mathrm{EM})}$ has very interesting theoretical properties. It has full gauge symmetry, but apparently describes "photons" propagating in media with velocities different from the velocity of light in vacuum. The "photons" in inhomogeneous media, therefore, have effective mass. The Lagrangian, if regarded as defining a truly quantum field-theoretic model, demonstrates, even at the classical "tree" level, that masslessness and gauge invariance have no necessary logical relationship. It should be of considerable interest to understand more fully the properties of the model.

Note added in proof. Our approach can also be applied to the model considered by Vollhardt and Wölfle [1] for electronic systems to reproduce the Ward identity they derived for charge conservation. In addition, there is in their model the conservation of energy, which implies another Ward identity similar to the ones we obtained in this paper. This is discussed by one of the authors elsewhere [20].

\section{ACKNOWLEDGMENTS}

We wish to thank Professor L. N. Chang for illuminating discussions. P.S. acknowledges the support of Hong Kong Research Grants Council Grant No. HKUST685/96P for this work.
[1] D. Vollhardt and P. Wölfle, Phys. Rev. B 22, 4666 (1980).

[2] See, e.g., B. A. van Tiggelen, Ph.D. thesis, FOM-Institute for Atomic and Molecular Physics, Amsterdam, Netherlands, 1992.

[3] B. A. van Tiggelen and A. Lagendijk, Europhys. Lett. 23, 311 (1993), and references therein.

[4] Yu. N. Barabanenkov and V. D. Ozrin, Phys. Lett. A 154, 38 (1991).

[5] Yu. N. Barabanenkov, L. M. Zurk, and M. Yu. Barabanenkov, J. Electron. Waves Appl. 9, 1393 (1995).

[6] Y. Takahashi, Nuovo Cimento 6, 370 (1957). A derivation of the Ward-Takahashi identity can be found in many textbooks on quantum field theory, e.g., S. Weinberg, The Quantum Theory of Fields (Cambridge University Press, Cambridge, 1995).

[7] J. C. Ward, Phys. Rev. 78, 182 (1950).

[8] See, e.g., M. Le Bellac, Quantum and Statistical Field Theory (Oxford Science Publications, Oxford, 1991).

[9] See, e.g., P. Sheng, Introduction to Wave Scattering, Localization, and Mesoscopic Phenomena (Academic, New York, 1995).

[10] M. P. van Albada, B. A. van Tiggelen, A. Lagenjijk, and A.
Tip, Phys. Rev. Lett. 66, 3132 (1991); B. A. van Tiggelen, A. Lagendijk, M. P. van Albada, and A. Tip, Phys. Rev. B 45, 12233 (1992).

[11] H. T. Nieh and P. Sheng, Phys. Lett. A 235, 259 (1997).

[12] See, e.g., W. Greiner and J. Reinhardt, Field Quantization (Springer-Verlag, Berlin, 1996).

[13] P. W. Anderson, Phys. Rev. 109, 1492 (1958).

[14] For reviews, see, e.g., Scattering and Localization of Classical Waves in Random Media, edited by P. Sheng (World Scientific, Singapore, 1990).

[15] See, e.g., U. Frisch, in Probabilistic Methods in Applied Mathematics, edited by A. T. Bharucha-Reid (Academic, New York, 1968), Vol. I.

[16] S. Engelsberg and J. R. Schrieffer, Phys. Rev. 131, 993 (1963).

[17] See, e.g., J. D. Bjorken and S. D. Drell, Relativistic Quantum Fields (McGraw-Hill, New York, 1965).

[18] G. Leibbrandt, Rev. Mod. Phys. 59, 1067 (1987), and references therein.

[19] We adopt a mixed notation for the three-dimensional Kronecker delta: $\delta_{j}^{i}=\delta_{i}^{j}=\delta^{i j}=\delta_{i j}=1$, if $i=j$, and zero otherwise.

[20] H. T. Nieh (unpublished). 